\title{
Topological charge density correlator in Lattice QCD with two flavours of unimproved Wilson fermions
}

\author{
Abhishek Chowdhury ${ }^{a}$, Asit K. De ${ }^{a}$, A. Harindranath ${ }^{a 1}$, Jyotirmoy Maiti ${ }^{b}$ and \\ Santanu Mondal ${ }^{a}$ \\ ${ }^{a}$ Theory Division, Saha Institute of Nuclear Physics \\ 1/AF Bidhan Nagar, Kolkata 700064, India \\ ${ }^{b}$ Department of Physics, Barasat Government College, \\ 10 KNC Road, Barasat, Kolkata 700124, India \\ E-mail: abhishek.chowdhury@saha.ac.in, asitk.de@saha.ac.in, \\ a.harindranath@saha.ac.in, jyotirmoy . maiti@gmail.com, \\ santanu.mondal@saha.ac.in
}

AbSTRACT: We study the two-point Topological Charge Density Correlator (TCDC) in lattice QCD with two degenerate flavours of unimproved Wilson fermions and Wilson gauge action at two values of lattice spacings and different volumes, for a range of quark masses. Configurations are generated with DDHMC algorithm and smoothed with HYP smearing. In order to shed light on the mechanisms leading to the observed suppression of topological susceptibility with respect to the decreasing quark mass and decreasing volume, in this work, we carry out a detailed study of the two-point TCDC. We have shown that, (1) the TCDC is negative beyond a positive core and radius of the core shrinks as lattice spacing decreases, (2) as the volume decreases, the magnitude of the contact term and the radius of the positive core decrease and the magnitude of the negative peak increases resulting in the suppression of the topological susceptibility as the volume decreases, (3) the contact term and radius of the positive core decrease with decreasing quark mass at a given lattice spacing and the negative peak increases with decreasing quark mass resulting in the suppression of the topological susceptibility with decreasing quark mass, (4) increasing levels of smearing suppresses the contact term and the negative peak keeping the susceptibility intact and (5) both the contact term and the negative peak diverge in nonintegrable fashion as lattice spacing decreases. It is gratifying to note that observations similar to 1 and 5 have been made using topological charge density operator based on chiral fermion. The observations 2 and 3 may be confirmed more precisely by using formulations based on chiral fermions.

\footnotetext{
${ }^{1}$ Corresponding Author
} 


\section{Contents}

1 Introduction 1

2 Measurements 2

3 Results 3

\section{Introduction}

As a consequence of the reflection positivity and the pseudoscalar nature of the relevant local operator in Euclidean quantum field theory, the two-point Topological Charge Density Correlator (TCDC) is negative at arbitrary non-zero distances [1]. In the continuum theory, close to the origin the two-point TCDC is negative and singular. From power counting, the singularity $\sim-|x|^{-8}$ up to possible logarithms and hence is non-integrable. In order to obtain a positive and finite space-time integral (susceptibility), the TCDC should have a positive non-integrable singularity at the origin $[1,2]$. However, it is possible to give a rigorous definition of topological susceptibility in Lattice QCD without power divergences using Ginsparg-Wilson fermion [3, 4].

As the authors of Ref. [1] pointed out long time ago, divergent behaviour of TCDC has nontrivial consequences for the derivation and interpretation of the Witten-Veneziano (WV) expression [5] for the $\eta^{\prime}$ mass. We note that the $\mathrm{WV}$ formula for the $\eta^{\prime}$ mass has been obtained later using the chiral Ward-Takahashi identity [3] which uses Ginsparg-Wilson fermion and gives an expression for topological susceptibility free from power divergences. The negativity of the TCDC also has non-trivial consequences related to the nature of topological charge structure in QCD vacuum [6].

The issues related to two-point TCDC are best studied in the theory rigorously formulated on a Euclidean lattice. However, the lattice theory defined by a particular action may not be reflection positive. Fortunately, this is not a concern for the Wilson fermion. However, the breaking of chiral symmetry by Wilson term may lead to uncancelled divergences in topological susceptibility. Thus it is important to calculate topological susceptibility with Wilson fermion to check whether the cancellation indeed happens so that Wilson lattice QCD belongs to the same universality class as continuum QCD.

The lattice operator for the topological charge density $q(x)$ may extend over several lattice spacings, and thus for sufficiently small $x$, the continuum like behaviors are not expected. Nevertheless, continuum properties are expected to emerge as lattice spacings become smaller and smaller. Specifically, on a lattice with lattice spacing $a$, TCDC remains positive within a radius $r_{c}$, which is expected to shrink to zero as $a \rightarrow 0$. The first investigation of lattice spacing dependence of the radius of the positive core and the negativity beyond the positive core of TCDC in lattice QCD was carried out in Ref. [7] in the context of overlap based topological charge density in quenched QCD. Later, similar study was carried out [8] for a variety of lattice QCD actions with and without quarks where discretization errors appear only at $\mathscr{O}\left(a^{2}\right)$. 


\begin{tabular}{|l|l|l|l|l|l|l|}
\hline tag & lattice & $\kappa$ & block & $N_{2}$ & $N_{\text {trj }}$ & $\tau$ \\
\hline$A_{2 b}$ & $16^{3} \times 32$ & 0.158 & $8^{4}$ & 10 & 6816 & 0.5 \\
\hline$B_{1 b}$ &,, & 0.1575 & $12^{2} \times 6^{2}$ & 18 & 13128 & 0.5 \\
$B_{3 b}$ &,, & 0.158 & $12^{2} \times 6^{2}$ & 18 & 13646 & 0.5 \\
$B_{4 b}$ &,, & 0.158125 & $12^{2} \times 6^{2}$ & 18 & 11328 & 0.5 \\
$B_{5 b}$ &,, & 0.15825 & $12^{2} \times 6^{2}$ & 18 & 12820 & 0.5 \\
\hline$C_{2}$ & $32^{3} \times 64$ & 0.158 & $8^{3} \times 16$ & 8 & 7576 & 0.5 \\
$C_{5}$ &,, & 0.1583 & $8^{3} \times 16$ & 8 & 11200 & 0.5 \\
\hline \hline \multicolumn{7}{|c|}{$\beta=5.8$} \\
\hline tag & lattice & $\kappa$ & block & $N_{2}$ & $N_{\text {trj }}$ & $\tau$ \\
\hline$D_{1}$ & $32^{3} \times 64$ & 0.1543 & $8^{3} \times 16$ & 8 & 9600 & 0.5 \\
$D_{3}$ &,, & 0.15462 & $8^{3} \times 16$ & 24 & 7776 & 0.5 \\
\hline \hline
\end{tabular}

Table 1. Lattice parameters and simulation statistics. Here block, $N_{2}, N_{t r j}$ and $\tau$ refer to HMC block, step number for the force $F_{2}$, number of HMC trajectories and the Molecular Dynamics trajectory length respectively.

Flavour singlet axial Ward-Takahashi identity relates the topological susceptibility $\chi$, which is the four-volume integral of TCDC, to the chiral condensate in the chiral limit $[9,10]$. As a consequence, $\chi$ vanishes linearly in the quark mass in the chiral limit. Furthermore, at a given value of the quark mass, $\chi$ is suppressed as volume decreases [10,11]. As part of an on-going program $[12,13]$ to study the chiral properties of Wilson lattice QCD (unimproved fermion and gauge actions), recently, we have demonstrated the suppression of topological susceptibility with decreasing quark mass in the case of unimproved Wilson fermion and gauge action [14, 15] where, the suppression of $\chi$ with decreasing volume was also shown. In order to shed light on the mechanisms leading to these suppressions, in this work, we carry out a detailed study of the two-point TCDC. Our interesting observations which are qualitative and suggestive at the moment may be confirmed more precisely by performing measurements at more values of lattice spacing and using chiral fermion formulations [3, 4].

\section{Measurements}

We have generated ensembles of gauge configurations by means of DDHMC algorithm [16] using unimproved Wilson fermion and gauge actions with $n_{f}=2$ mass degenerate quark flavours. At $\beta=5.6$ the lattice volumes are $16^{3} \times 32,24^{3} \times 48$ and $32^{3} \times 64$ and the renormalized quark mass ranges between 25 to $125 \mathrm{MeV}$ ( $\overline{\mathrm{MS}}$ scheme at $2 \mathrm{GeV}$ ). At $\beta=5.8$ the lattice volume is $32^{3} \times 64$ and the renormalized physical quark mass ranges from 15 to $75 \mathrm{MeV}$. The lattice spacings are determined using nucleon mass to pion mass ratio and Sommer method. These determinations agree for the value of Sommer parameter $r_{0}=0.44 \mathrm{fm}$. The lattice spacings at $\beta=5.6$ and 5.8 are 0.069 and $0.053 \mathrm{fm}$ respectively. The number of thermalized configurations ranges from 7000 to 
14000 and the number of measured configurations ranges from 200 to 500. Parameters for a subset of our runs that are used in this paper are given in Table 1.

The topological susceptibility

$$
\chi=\int d^{4} x C(r)
$$

with the TCDC,

$$
C(r)=\langle q(x) q(0)\rangle, \quad r=|x|
$$

where $q(x)$ is the topological charge density.

From the definition of the topological susceptibility

$$
\chi=\int d^{4} x C(r)=\int 2 \pi^{2}\left(r^{3}\right) d r C(r)
$$

it is useful to define [17] a local susceptibility

$$
\chi(r)=\int_{0}^{r} 2 \pi^{2}\left(r^{\prime 3}\right) d r^{\prime} C\left(r^{\prime}\right)
$$

in order to exhibit the lattice spacing dependence more clearly. It is also useful to define the contributions to the susceptibility from the positive and negative parts of $C(r)$ as

$$
\begin{aligned}
& \chi_{P}=\int_{0}^{r_{c}} 2 \pi^{2}\left(r^{\prime 3}\right) d r^{\prime} C\left(r^{\prime}\right) \text { and } \\
& \chi_{N}=\int_{r_{c}}^{\infty} 2 \pi^{2}\left(r^{\prime 3}\right) d r^{\prime} C\left(r^{\prime}\right)
\end{aligned}
$$

respectively. For $q(x)$, we use the lattice approximation developed for $S U(2)$ by DeGrand, Hasenfratz and Kovacs [18], modified for $S U(3)$ by Hasenfratz and Neiter [19] and implemented in the MILC code [20]. It uses ten link paths described by unit lattice vector displacements in the sequence $\{x, y, z,-y,-x, t, x,-t,-x,-z\}$ and $\{x, y, z,-x, t,-z, x,-t,-x,-y\}$ plus rotations and cyclic permutations. We used HYP smearing with optimized smearing coefficients $\alpha=0.75$, $\alpha_{2}=0.6$ and $\alpha_{3}=0.3$ [21]. Unless otherwise stated we have used 3 smearing steps in all our calculations.

\section{Results}

In order to extract the topological charge density reliably on the lattice, using the algebraic definition, smearing of link field is essential. Smearing however smoothens out short distance singularities. Excessive smearing may in fact wipe out the fine details of the singularity structure. Both the positive and negative contributions to $\chi$ are affected in this manner. This is illustrated in Fig. 1 where we show the effect of 3, 5, 10 and 20 HYP smearing steps on $C(r)$ at $\beta=5.8, \kappa=0.15462$ and lattice volume $32^{3} \times 64$. However the susceptibility is remarkably stable under smearing after three smearing steps as illustrated in Fig. 2 (taken from Ref. [15]).

As was already stated, from theoretical considerations, we expect suppression of $\chi$ with decreasing volume at a fixed quark mass. In Fig. 3 we present the finite volume dependence of the 


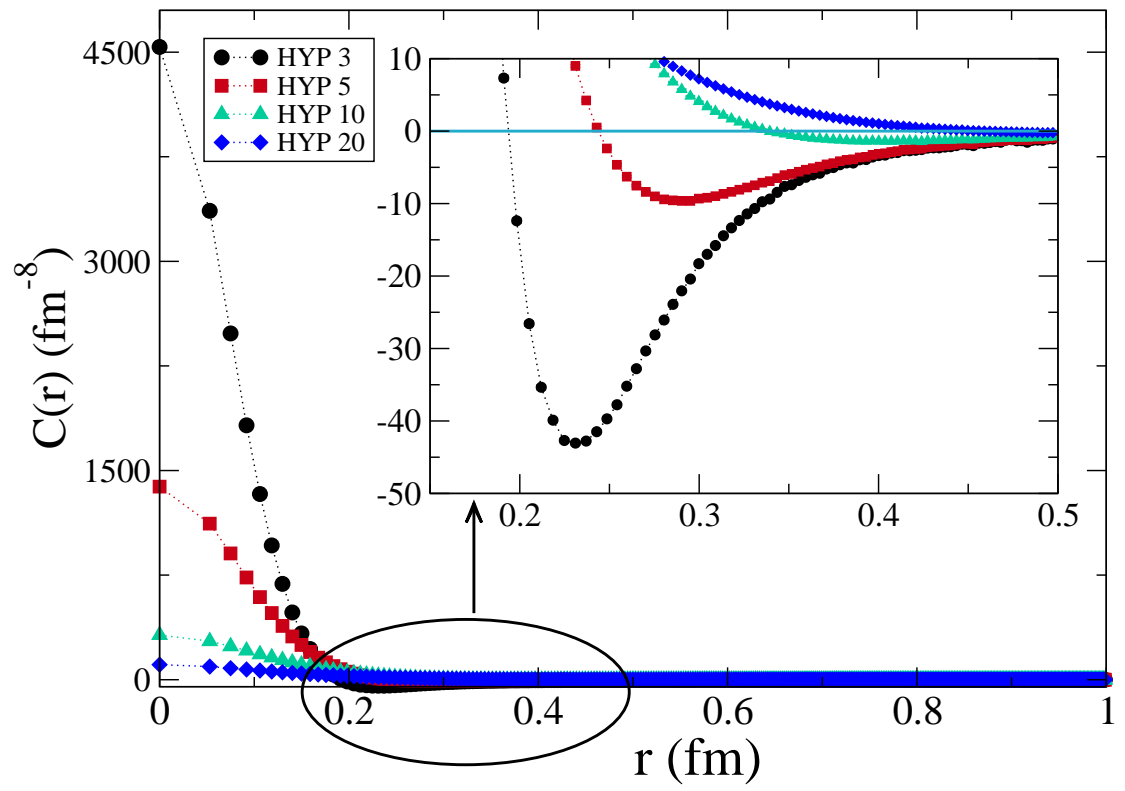

Figure 1. Effect of smearing on $C(r)$ at $\beta=5.8, \kappa=0.15462$ and lattice volume $32^{3} \times 64$.

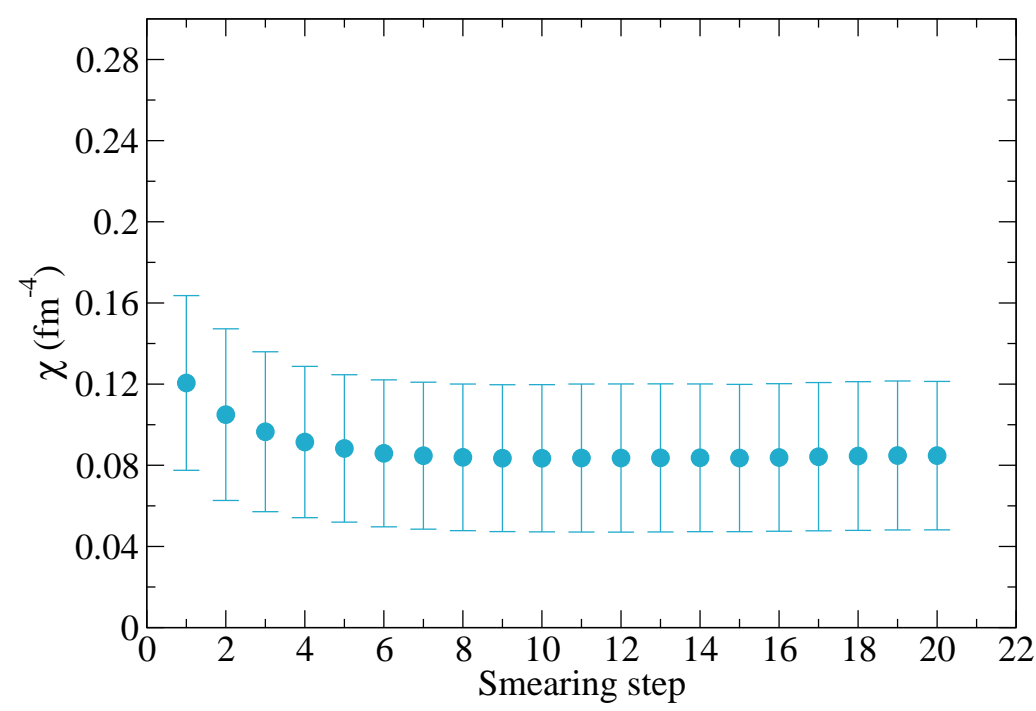

Figure 2. Effect of smearing on the $\chi$ at $\beta=5.8, \kappa=0.15462$ and lattice volume $32^{3} \times 64$ (taken from Ref. [15]). 


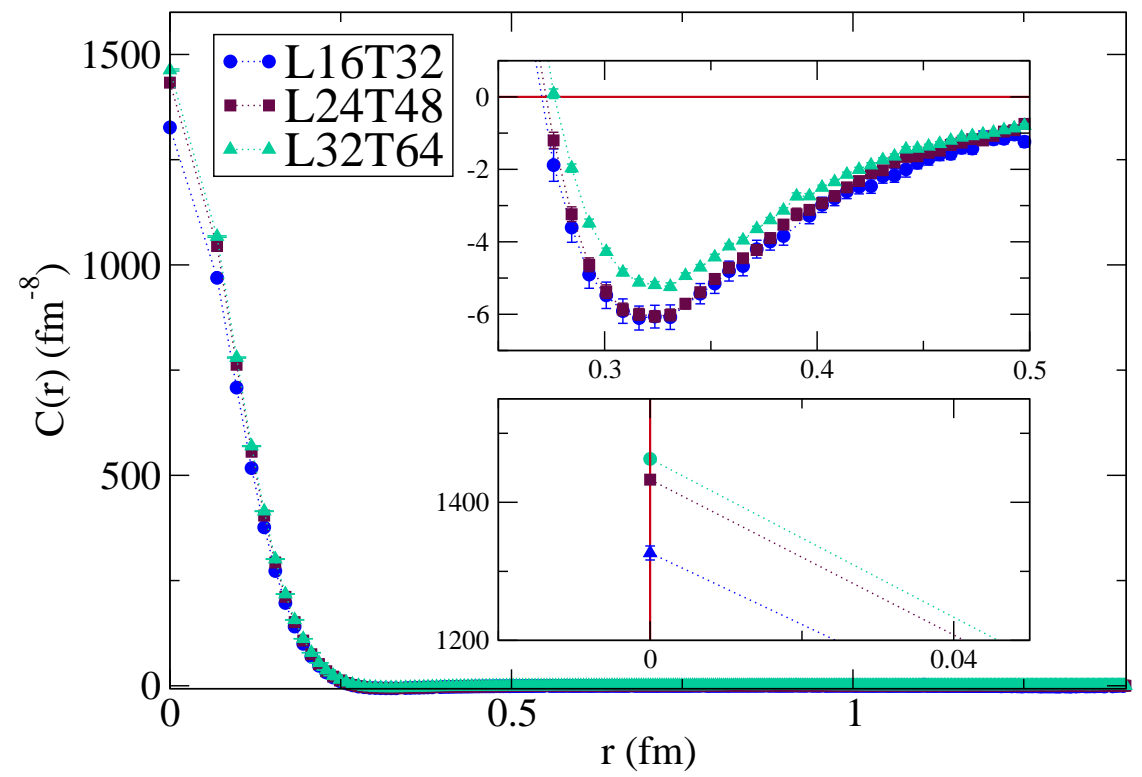

Figure 3. Finite volume dependence of the $C(r)$ at $\beta=5.6, \kappa=0.158$ and lattice volumes $16^{3} \times 32,24^{3} \times 48$ and $32^{3} \times 64$.

$C(r)$ at $\beta=5.6$ and $\kappa=0.158$ at lattice volumes $16^{3} \times 32,24^{3} \times 48$ and $32^{3} \times 64$. We find that as volume decreases, the magnitude of the contact term and radius of the positive core decrease and the magnitude of the negative peak increases resulting in the suppression of topological susceptibility as volume decreases.

In order to understand the detailed mechanism behind the suppression of topological susceptibility with decreasing quark mass, we need to investigate the quark mass dependence of the various features of the $C(r)$. In Fig. 4 we present the quark mass dependence of $C(r)$ with emphasis on the positive region at $\beta=5.6$ and lattice volume $24^{3} \times 48$. The magnitude of the contact term $C(0)$ is seen to decrease with decreasing quark mass. In Fig. 5 we present the quark mass dependence of $C(r)$ with emphasis on the crossover from positive to the negative region of $C(r)$ and the negative peak region at $\beta=5.6$ and lattice volume $24^{3} \times 48$. The radius of the positive core and the magnitude of the negative peak of $C(r)$ are seen to decrease and increase respectively with decreasing quark mass. The features presented in Figs. 4 and 5 result in the suppression of the topological susceptibility with decreasing quark mass. MILC collaboration [17] has made a similar observation regarding the dependence of the negative peak on quark mass. In Fig. 6 we present the corresponding topological susceptibilities ( $\beta=5$.6, lattice volume $24^{3} \times 48$ and smearing step $20)$ as a function of the quark mass which clearly shows the suppression as quark mass decreases. This figure includes $\kappa=0.158$ in addition to the $\kappa$ 's presented in Figs. 4 and 5.

In Table 2 we show the lattice spacing dependence of the contact term $C(0)$ and the magnitude of the negative peak of $C(r)\left(\left|C^{\min }\right|\right)$ at comparable pion mass in physical units for $\beta=5.6$ and 


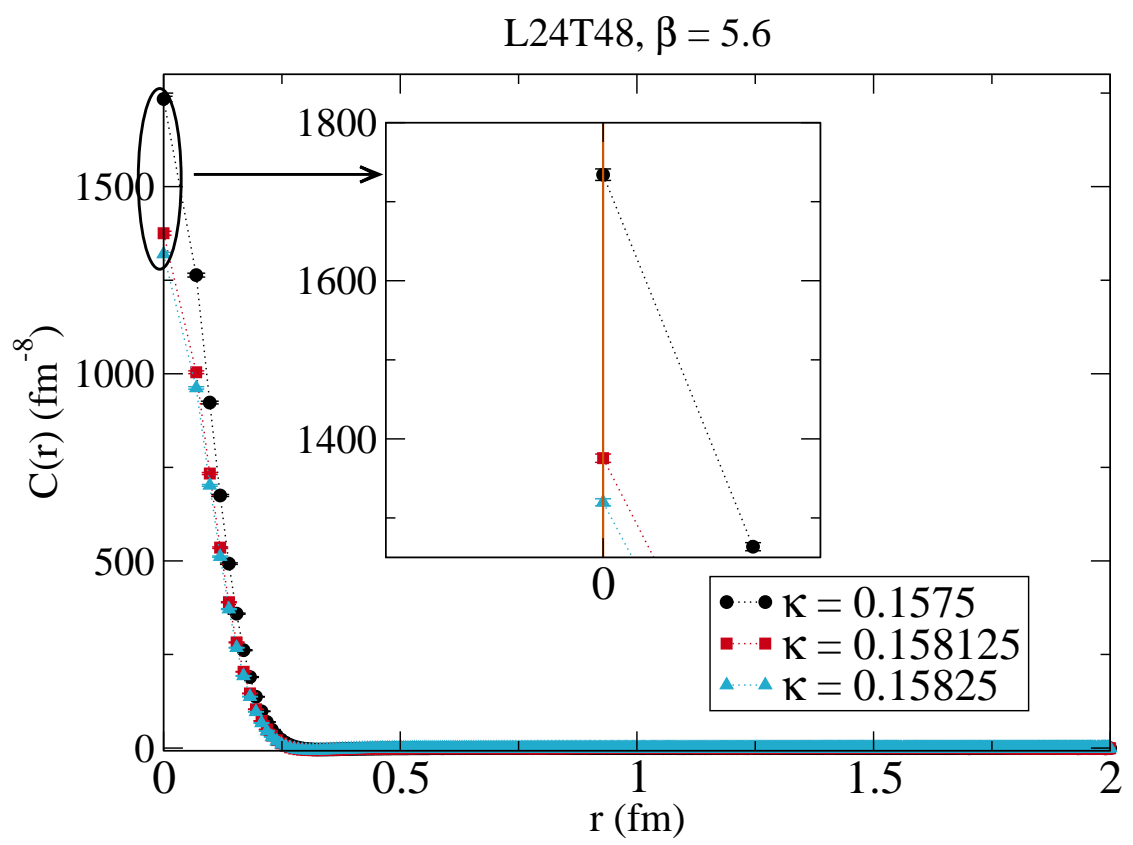

Figure 4. The quark mass dependence of $C(r)$ with emphasis on the positive region at $\beta=5.6$ and lattice volume $24^{3} \times 48$.

\begin{tabular}{|l|l|l|l|l|}
\hline \hline tag & $\begin{array}{l}a \\
(\mathrm{fm})\end{array}$ & $\frac{r_{c}}{a}$ & $\begin{array}{l}C(0) \\
\left(\mathrm{fm}^{-8}\right)\end{array}$ & $\begin{array}{l}\left|C^{\text {min }}\right| \\
\left(\mathrm{fm}^{-8}\right)\end{array}$ \\
\hline$C_{5}$ & 0.069 & 3.93 & $1336(3)$ & $5.8(1)$ \\
$D_{3}$ & 0.053 & 3.65 & $4537(7)$ & $43.0(3)$ \\
\hline \hline
\end{tabular}

Table 2. Lattice spacing dependence of $\frac{r_{c}}{a}, C(0)$ and $\left|C^{\text {min }}\right|$ at comparable pion mass.

\begin{tabular}{|l|l|l|l|l|}
\hline \hline tag & $\begin{array}{l}a \\
(\mathrm{fm})\end{array}$ & $\begin{array}{l}\chi_{P} \\
\left(\mathrm{fm}^{-4}\right)\end{array}$ & $\begin{array}{l}\chi_{N} \\
\left(\mathrm{fm}^{-4}\right)\end{array}$ & $\begin{array}{l}\chi \\
\left(\mathrm{fm}^{-4}\right)\end{array}$ \\
\hline$B_{3 b}$ & 0.069 & 2.85 & 1.61 & 1.24 \\
$D_{1}$ & 0.053 & 3.27 & 2.19 & 1.08 \\
\hline \hline
\end{tabular}

Table 3. Lattice spacing dependence of $\chi_{P}, \chi_{N}$ and $\chi$ at comparable pion mass.

5.8 and lattice volume $32^{3} \times 64$. For comparison, the corresponding quantities for pure gauge lattice theory at $\beta=6.0983(a=0.078 \mathrm{fm})$ and lattice volume $24^{3} \times 48$ are $C(0)=285(1)\left(\mathrm{fm}^{-8}\right)$ and $\left|C^{\min }\right|=0.69(5)\left(\mathrm{fm}^{-8}\right)$. Both the contact term and the negative peak of $C(r)$ increase with decreasing lattice spacing, in accordance with the expectation from the continuum theory. 


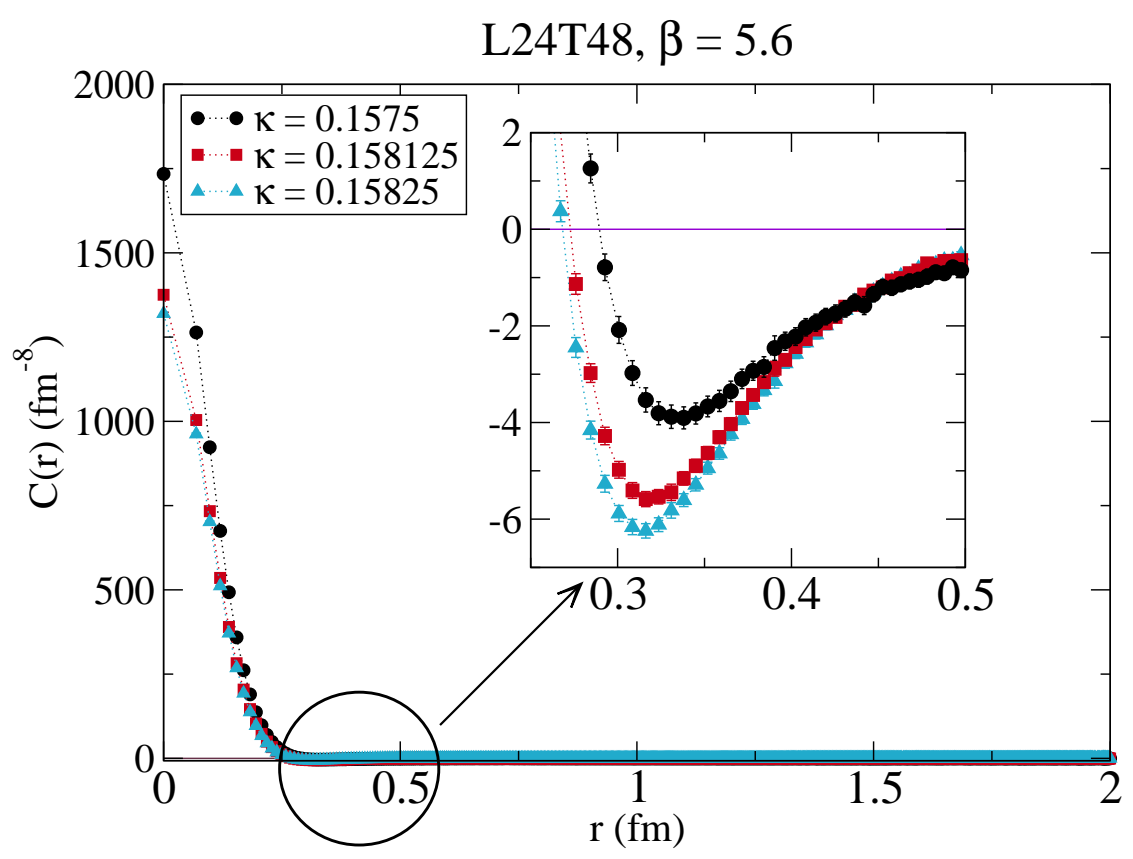

Figure 5. The quark mass dependence of $C(r)$ with emphasis on the crossover from positive to the negative region of $C(r)$ and the negative peak region at $\beta=5.6$ and lattice volume $24^{3} \times 48$.

In Fig. 7 we plot $\chi(r)$ versus $r$ at two lattice spacings at comparable quark masses. The contribution from the positive part of $C(r)$ results in a peak at short distance. This is followed by a decrease due to the negative part of $C(r)$. As lattice spacing decreases, the contribution from the positive part increases resulting in the increase of the peak of $\chi(r)$.

According to the expectations from continuum theory, the negative singularity close to the origin and the positive singularity at the origin are both nonintegrable. Thus the contributions to $\chi$ from positive and negative parts of $C(r)$ are expected to diverge, nevertheless resulting in a finite $\chi$ due to cancellation. In Table 3, we show the contributions to the susceptibility from positive and negative parts of $C(r)$ at $\beta=5.6$ and 5.8 at comparable pion masses. The data shown in Table 3 are in accordance with these expectations.

In Fig. 8, we compare the radius of the positive core of $C(r)$ at $\beta=5.6$ and 5.8 for comparable pion masses in physical units. The lattice volume is $32^{3} \times 64$. The figure clearly exhibits the shrinking of the radius of the positive core of $C(r)$ in physical units as one approaches the continuum.

It is gratifying to note that the various trends regarding lattice spacing dependence shown in Tables 2 and 3 and Fig. 8 have also been observed [7] by using topological charge density operator based on Ginsparg-Wilson fermion [3].

It is known that the topological susceptibility decreases with decreasing quark mass and decreasing volume. This has also been demonstrated $[14,15]$ using Wilson Lattice QCD which has $\mathscr{O}(a)$ lattice artifacts. To understand the mechanisms leading to these suppressions, in this work, 


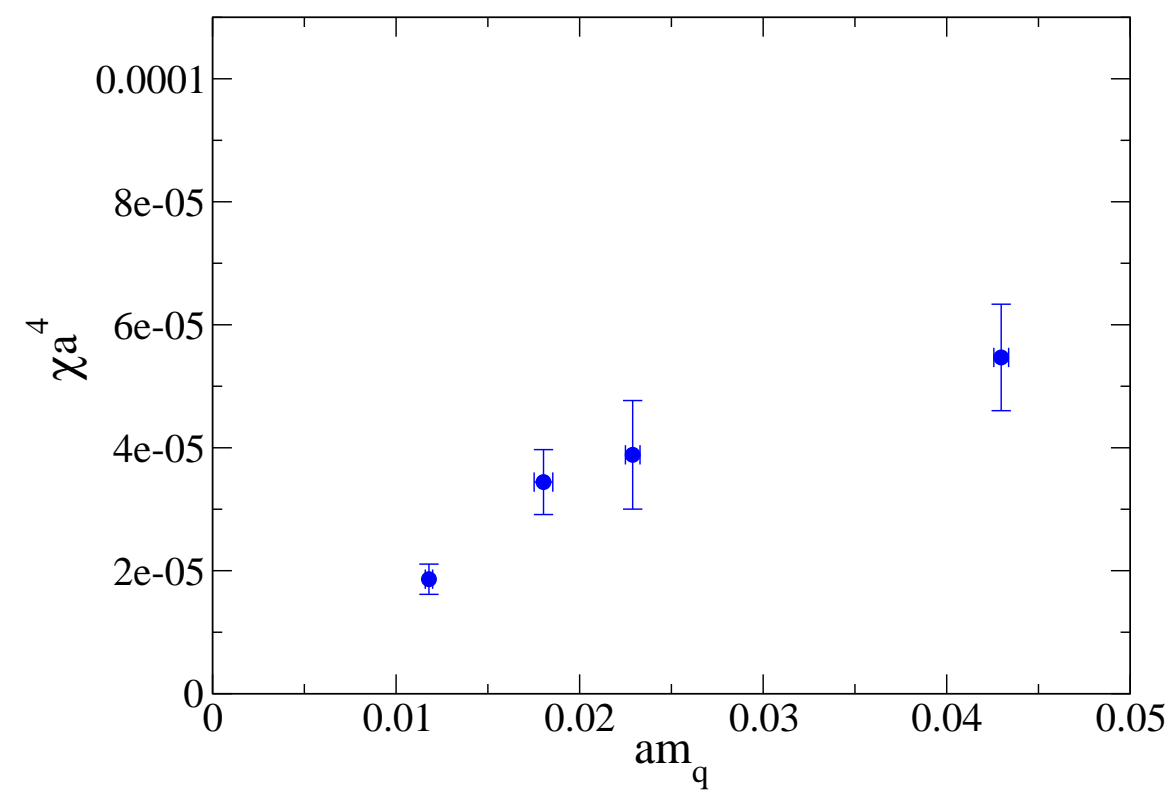

Figure 6. Topological susceptibility at $\beta=5.6$ and lattice volume $24^{3} \times 48$ as a function of the quark mass mass.

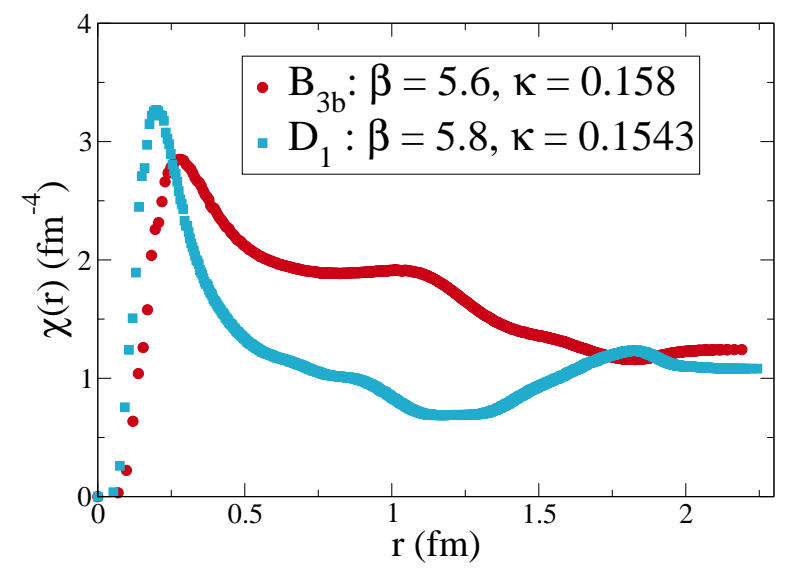

Figure 7. The function $\chi(r)$, defined in Eq. (2.3) as a function of $r$ at $\beta=5.6$ and 5.8 at comparable pion masses.

we carry out a detailed study of the two-point TCDC. We have shown that, with unimproved Wilson fermions and Wilson gauge action, (1) the two-point TCDC is negative beyond a positive core and radius of the core shrinks as lattice spacing decreases, (2) as volume decreases, the magnitude of the contact term and the radius of the positive core decrease and the magnitude of the nega- 


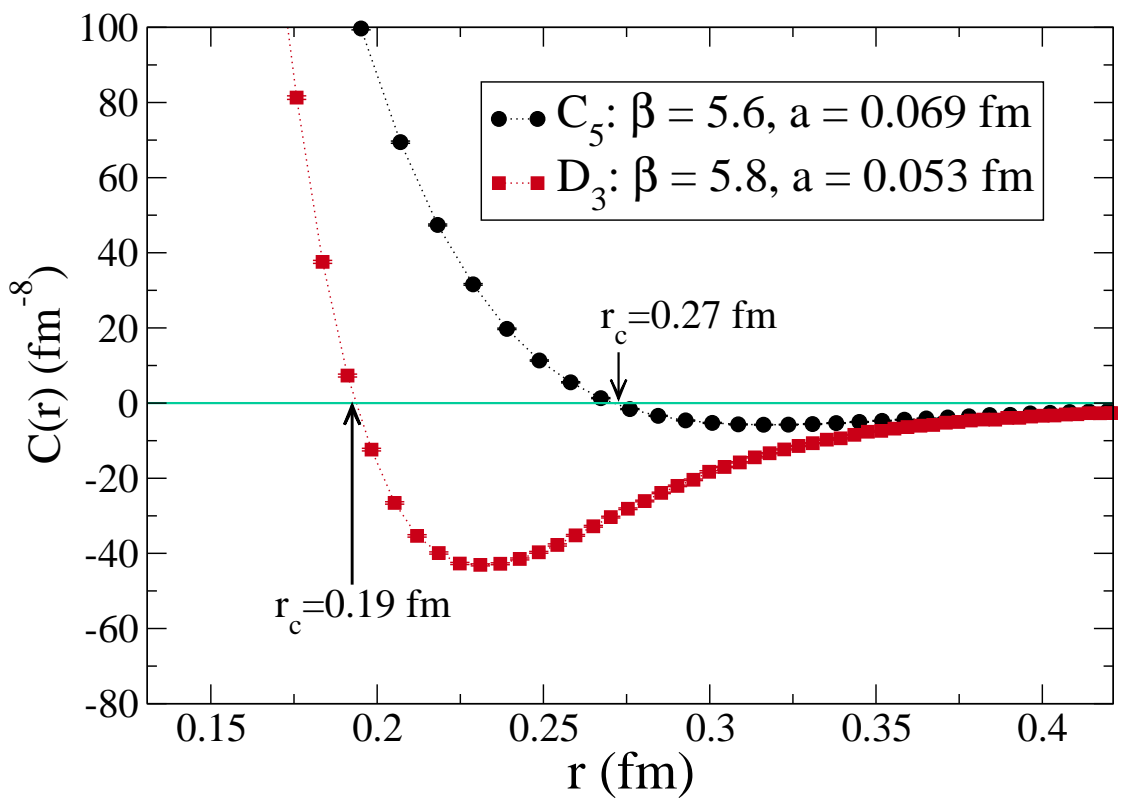

Figure 8. Comparison of the radius of the positive core of $C(r)$ at two different lattice spacings for comparable pion mass. Lattice volume is $32^{3} \times 64$.

tive peak increases resulting in the suppression of topological susceptibility as volume decreases, (3) the contact term and radius of the positive core decrease with decreasing quark mass at a given lattice spacing and the negative peak increases with decreasing quark mass resulting in the suppression of the topological susceptibility with decreasing quark mass, (4) increasing levels of smearing suppresses the contact term and the negative peak keeping the susceptibility intact and (5) both the contact term and the negative peak diverge in nonintegrable fashion as lattice spacing decreases. Observations similar to 1 and 5 have been made using topological charge density operator based on Ginsparg-Wilson fermion.

The observations 2 and 3 need to be confirmed by using formulations based on chiral fermions. For a quantitative determination of the nature of divergences noted in observation 5, it is necessary to have measurements at more values of lattice spacing. Our results for topological susceptibilities at two lattice spacings presented in Ref. [14] clearly show scaling violation. We note that a satisfactory definition $[3,4]$ of topological susceptibility using Ginsparg-Wilson fermion exhibits properties as in the continuum and avoids potential ultra-violet lattice artifacts present in TCDC when naive lattice operators are used.

\section{Acknowledgements}

Numerical calculations are carried out on Cray XD1 and Cray XT5 systems supported by the 10th and 11th Five Year Plan Projects of the Theory Division, SINP under the DAE, Govt. of 
India. We thank Richard Chang for the prompt maintainance of the systems and the help in data management. This work was in part based on the public lattice gauge theory codes of the MILC collaboration [20] and Martin Lüscher [16].

\section{References}

[1] E. Seiler and I. O. Stamatescu, Some remarks on the Witten-Veneziano formula for the Eta-prime mass, MPI-PAE/PTh 10/87, unpublished; E. Seiler, Phys. Lett. B 525, 355 (2002).

[2] M. Aguado and E. Seiler, Phys. Rev. D 72, 094502 (2005) [hep-lat/0503015].

[3] L. Giusti, G. C. Rossi and M. Testa, Phys. Lett. B 587, 157 (2004) [hep-lat/0402027]. See also L. Giusti, G. C. Rossi, M. Testa and G. Veneziano, Nucl. Phys. B 628, 234 (2002) [hep-lat/0108009].

[4] M. Luscher, Phys. Lett. B 593, 296 (2004) [hep-th/0404034].

[5] E. Witten, Nucl. Phys. B 156, 269 (1979); G. Veneziano, Nucl. Phys. B 159, 213 (1979).

[6] I. Horvath, S. J. Dong, T. Draper, F. X. Lee, K. F. Liu, N. Mathur, H. B. Thacker and J. B. Zhang, Phys. Rev. D 68, 114505 (2003) [hep-lat/0302009].

[7] I. Horvath, A. Alexandru, J. B. Zhang, Y. Chen, S. J. Dong, T. Draper, K. F. Liu, N. Mathur, S. Tamhankar and H. B. Thacker, Phys. Lett. B 617, 49 (2005) [hep-lat/0504005].

[8] F. Bruckmann, F. Gruber, N. Cundy, A. Schafer and T. Lippert, Phys. Lett. B 707, 278 (2012) [arXiv:1107.0897 [hep-lat]].

[9] R. J. Crewther, Phys. Lett. B 70, 349 (1977).

[10] H. Leutwyler and A. V. Smilga, Phys. Rev. D 46, 5607 (1992).

[11] For a detailed discussion, see S. Durr, Nucl. Phys. B 611, 281 (2001) [hep-lat/0103011].

[12] A. K. De, A. Harindranath and S. Mondal, Phys. Lett. B 682, 150 (2009) [arXiv:0910.5611 [hep-lat]].

[13] A. K. De, A. Harindranath and S. Mondal, JHEP 1107, 117 (2011) [arXiv:1105.0762 [hep-lat]].

[14] A. Chowdhury, A. K. De, S. De Sarkar, A. Harindranath, S. Mondal, A. Sarkar and J. Maiti, Phys. Lett. B 707, 228 (2012) [arXiv:1110.6013 [hep-lat]].

[15] A. Chowdhury, A. K. De, S. De Sarkar, A. Harindranath, S. Mondal, A. Sarkar and J. Maiti, PoS LATTICE 2011, 099 (2011) [arXiv:1111.1812 [hep-lat]].

[16] M. Lüscher, Comput. Phys. Commun. 156, 209-220 (2004). [hep-lat/0310048]; M. Lüscher, Comput. Phys. Commun. 165, 199-220 (2005). [hep-lat/0409106]. http://luscher.web.cern.ch/luscher/DD-HMC/index.html

[17] A. Bazavov et al. [MILC Collaboration], Phys. Rev. D 81, 114501 (2010) [arXiv:1003.5695 [hep-lat]].

[18] T. A. DeGrand, A. Hasenfratz, T. G. Kovacs, Nucl. Phys. B505, 417-441 (1997). [arXiv:hep-lat/9705009 [hep-lat]].

[19] A. Hasenfratz, C. Nieter, Phys. Lett. B439, 366-372 (1998). [hep-lat/9806026].

[20] http://physics.indiana.edu/ sg/milc.html

[21] A. Hasenfratz, F. Knechtli, Phys. Rev. D64, 034504 (2001). [hep-lat/0103029]. 